Published in final edited form as:

Eur J Cancer. 2019 May ; 112: 118-126. doi:10.1016/j.ejca.2018.11.033.

\title{
Prostate Cancer Incidence and Mortality among Men using Statins and Non-statin Lipid-lowering Medications
}

\author{
Maria I. Van Rompay, PhD ${ }^{\# a}$, Keith R. Solomon, PhD $^{\# b}$, J. Curtis Nickel, MD $^{c}$, Gayatri \\ Ranganathan, MS ${ }^{\mathrm{a}}$, Philip W. Kantoff, MD ${ }^{\mathrm{d}}$, and John B. McKinlay, PhD ${ }^{\mathrm{a}, \mathrm{e}}$ \\ aHealthCore-NERI, 480 Pleasant Street, Watertown, MA 02472 USA \\ bDepartment of Orthopaedic Surgery, Harvard Medical School, and the Departments of \\ Orthopaedic Surgery and Urology, Boston Children's Hospital, Boston, MA 02115 USA. Present \\ address: Applied Photophysics, 100 Cummings Center, Suite 440C, Beverly, MA 01915 USA \\ 'Queen's University and Kingston General Hospital, Kingston, Ontario, Canada \\ dMemorial Sloan Kettering Cancer Center and Weill Cornell Medical College, New York, NY \\ 10065 USA \\ eMassachusetts General Hospital, Harvard Medical School, Boston, MA 02114 USA \\ \# These authors contributed equally to this work.
}

\begin{abstract}
Background: Statins have demonstrated protection against aggressive/late-stage and/or lethal prostate cancer (PC), but prior studies are limited by small populations, short follow-up, and unequal health care access. Research has not demonstrated that non-statin lipid-lowering medications (NSLLM) provide a similar benefit, which would support a cholesterol-based mechanism. We sought to rigorously test the hypothesis that cholesterol-lowering drugs affect PC incidence and severity.
\end{abstract}

Methods: A retrospective cohort study was conducted by abstracting prescription and health service records for 249,986 Saskatchewan men aged $\geq 40$ years between January 1,1990 and December 31, 2014, and comparing first-time statin and NSLLM users with age-matched non-

\footnotetext{
Corresponding Author: Maria I. Van Rompay, PhD, HealthCore-NERI, 480 Pleasant Street, Suite A100, Watertown, MA 02472 USA, Tel: +1 (617) 972-3281; Fax: +1 (617) 673-9547; mvanrompay@ neriscience.com.

Publisher's Disclaimer: This is a PDF file of an unedited manuscript that has been accepted for publication. As a service to our customers we are providing this early version of the manuscript. The manuscript will undergo copyediting, typesetting, and review of the resulting proof before it is published in its final citable form. Please note that during the production process errors may be discovered which could affect the content, and all legal disclaimers that apply to the journal pertain.

Conflict of interest statement:

Dr. Nickel reports that GlaxoSmithKline provided him and his collaborators with the database from the dutasteride REDUCE study with no associated financial considerations or publication restrictions. Dr. Kantoff reports personal fees from Astellas, Bayer, Bellicum, BIND Biosciences, Bavarian Nordic Immunotherpeutics, DRGT, Genetech/Roche, Ipsen Pharmaceuticals, Janssen, Metamark, Merck, Millennium/Prometrika, MTG, Omnitura, OncoCellMDX, OncoGenex, Progenity, Sanofi, Tarveda Therapeutics (formerly Blend), Thermo Fisher, GE Healthcare, Context Therapeutics, and SEER Biosciences, outside the submitted work; in addition, Dr. Kantoff reports an investment interest from DRGT, Tarveda Therapeutics (formerly Blend), Context Therapeutics, SEER Biosciences, and Placon, outside the submitted work, and he reports being a company board member of Context Therapeutics, outside the submitted work. All other authors have no competing interests.
} 
users and glaucoma medication (GM) users for PC incidence, metastases at diagnosis, and PC mortality using Cox proportional hazards regression.

Results: In comparing statin users to non-users, a weak association was detected with increased PC incidence (HR 1.07, 95\% CI: 1.02-1.12) that disappeared when compared with GM users. Substantial protective associations were observed between statin use and metastatic PC and PC mortality (HRs 0.69, 95\% CI: 0.61-0.79 and 0.73, 95\% CI: 0 66-0.81, respectively), which were stronger when compared with GM use (HRs $0.52,95 \%$ CI: 0.40-0.68 and 0.51, 95\% CI: 0.41-0.63, respectively). Similar associations were found for NSLLM vs. GM: for metastatic PC, HR 0.57, 95\% CI: 0.41-0.79 and PC mortality, 0.66, 95\% CI: 0.51-0.85.

Conclusions: Our analyses provide one of the more comprehensive findings to date that statins may reduce risk of metastatic PC and PC mortality, and the first to demonstrate that NSLLM have similar effects, supporting a cholesterol-based mechanism.

\section{Keywords}

Hydroxymethylglutaryl-CoA Reductase Inhibitors; Prostatic Neoplasms; pharmacoepidemiology

\section{Introduction}

Statins (3-hydroxy-3-methylglutaryl coenzyme A reductase inhibitors) are widely prescribed drugs used to reduce low density lipoprotein (LDL) cholesterol and modulate risk of cardiovascular disease (CVD). Although initially intended to treat individuals with higher comparative CVD risk [1], they have been shown to reduce CVD in patients with low or moderate risk [2], with the consequence that patients without atherosclerosis or other prominent CVD risk factors have been prescribed statins for many years. This prescribing practice allows for retrospective analysis of large patient populations to evaluate possible effects of statins on risk of non-cardiovascular diseases, including prostate cancer (PC), a malignancy that has been experimentally and epidemiologically linked in several reports to cholesterol [3-7].

Prior studies investigating statins and PC incidence are somewhat equivocal. Large randomized trials of statins do not support a protective role on PC risk [8,9]. Most $[4,10$ 17], but not all [18-20], observational studies suggest that, while statins may have a small effect on PC incidence, they potentially have a substantial effect on PC severity, an interpretation supported by meta-analyses [17,21]. Observational studies suggest a compelling protective association, but many are weakened by limitations. When comparing statin users with non-users, a statin effect on PC risk may be due to an extraneous difference such as a patient's health care access or utilization, a concern addressed by having another drug user group as the comparison [12,22]. Relatively short follow-up [12,14,16] may preclude evidence for a protective statin effect if cancers have not reached clinical detection by study's end. Moreover, small study populations $[18,19]$ and reliance on "soft" endpoints such as Gleason score $[10,14,23]$ invite subjectivity and often much missing data. Furthermore, given that we expect the primary mechanism of any patient-relevant statin effect on PC disease risk to be through LDL reduction [3-7], prior studies have not 
considered this mechanism by examining whether other cholesterol-lowering drugs also have an effect, although one prior analysis of NSLLM on PC risk found no association [15].

To test the hypothesis that cholesterol-lowering drugs affect incidence of overall PC, metastatic PC at diagnosis, and PC mortality, and address shortcomings of prior analyses $[20,24]$, we performed a population-based study of men aged $\geq 40$ years from Canada's Saskatchewan province for their first-time statin, NSLLM, and glaucoma medication (GM) prescriptions over 25 years, and compared PC incidence and mortality endpoints, as well as metastatic PC, between statin users and non-users, NSLLM users and non-users, and statin or NSLLM users and GM users.

\section{Patients and methods}

\subsection{Data source and population}

The Saskatchewan Ministry of Health compiled de-identified data for 249,986 men aged $\geq 40$ years covered by Saskatchewan Health $(\mathrm{SH})$ and eligible for provincial outpatient prescription drug benefits between January 1, 1990 and December 31, 2014. Based on predefined criteria (detailed in section 2.2), the dataset included a subset of the men's outpatient prescription drug, medical services, and health insurance registration records, as well as certain cancer records from the Saskatchewan Cancer Agency (SCA) cancer registry. The study was approved by the institutional review board of HealthCore-NERI, Watertown, MA, and the University of Saskatchewan Biomedical Research Ethics Board.

\subsection{Drug exposure groups}

We identified new users of statins, NSLLM, and GM among men aged $\geq 40$ years in Saskatchewan. Index date was defined as dispensing date of the first prescription on or after the man's $40^{\text {th }}$ birthday, no history of cancer prior to index, and study entry as 365 days prior to index to ensure that participants were covered by SH for $\geq 12$ months and that the first prescription in the database was their first. Specific drugs of interest are listed in Supplemental Table 1. For each statin user, up to three non-users matched on age (up to \pm 2 years) were selected; for each NSLLM user, up to ten matched non-users were selected, with a higher ratio of NSLLM non-users chosen to enable sufficient power. Non-users reported the same index date as their matched user, and a statin user or NSLLM user may have served as a non-user prior to receiving his first index prescription. As an active comparator, GM users controlled for confounding and reduced bias related to differential health care exposure and prescribing patterns common to preventive therapy medications for potentially asymptomatic chronic health conditions [25-27]. Because the statin/NSLLM user groups and GM group had drug treatment initiation dates, these dates served as easily identifiable, meaningful start times for collecting baseline characteristics and starting follow-up [26,27]. Furthermore, having an active comparator reduced the potential for confounding by frailty that could occur with a "non-user" older adult group, where frail or sick individuals may not be prescribed preventive treatments related to failing health [27]. 


\subsection{Outcomes}

Incident PCs were identified from SCA registry data using first occurrence date of ICD-O-3 code C61.9 for primary prostate carcinoma, limited to adenocarcinomas. PC mortality was obtained from SCA registry data on cause of death. To define more advanced PC, metastases at diagnosis was used.

\subsection{Covariates}

Covariates of interest were age at index, index year, baseline diabetes, and baseline use of other drugs of interest, including other LLM, a blockers, and 5a-reductase inhibitors (5ARI), with "baseline" defined as within 12 months prior to index through the index date. Physician services records provided data on certain medical diagnoses of interest; however, only one diagnosis was reported for each medical visit. For analysis, index year was initially categorized into five groups of quinquennia; however, due to small numbers in the first and second groups, four categories of index year were used: 1990-1999 (reference), 2000-2004, 2005-2009, and 2010-2014.

\subsection{Statistical analyses}

Descriptive analyses compared demographic and clinical characteristics of male new users of statins, NSLLM, their respective non-users, and GM users. A retrospective cohort study design using survival analysis methods, including log rank statistics and Cox proportional hazards models, quantified differences in incident PC, metastatic PC, and PC mortality between drug exposure groups. Exit date (censoring date) was defined as the earliest of date of cancer diagnosis, coverage termination with SH, death, or December 31, 2014, in that order, and follow-up time (person-time) as the time between index and exit date. For each outcome, two regression models were run: basic models adjusting only for age at index, and multivariable models also adjusting for index year, baseline diabetes, and baseline use of ablockers, 5ARI, and other LLM. For all Cox models, the proportional-hazards assumption was tested with PROC PHREG. Statistical analyses were performed using SAS 9.4 (SAS Institute, Inc.), with 2-tailed tests and $\mathrm{P}$ values $<0.05$ representing statistical significance.

\section{Results}

Across new users of statins, NSLLM, and GM, as well as non-users, median (IQR) followup time ranged from 5.9 (7.0) years among statin non-users, to 7.3 (8.8) years among GM users (Tables 1 and 2). Statin users had a longer duration of follow-up than their agematched non-users, and GM users were older at index. Statin users and NSLLM users were more likely to use other LLM at baseline, and to have cardiometabolic conditions than nonusers. Moreover, while statin and NSLLM users had lower baseline prevalence of benign prostatic hyperplasia than GM users, they were more likely to have been prescribed 5ARIs or a-blockers.

Cumulative incidence of PC was 3.9\% in statin users, $3.1 \%$ in statin non-users, $4.2 \%$ in NSLLM users, 3.9\% in NSLLM non-users, and 5.7\% in GM users (Tables 1 and 2). Personyears of observation for each group are provided in Supplemental Table 2. In multivariableadjusted survival analyses, compared with non-users, statin users and NSLLM users had a 
$7 \%$ and $14 \%$ higher risk of PC incidence, respectively; however, when compared with GM users, there were no associations with PC incidence (Table 3). For metastatic PC, both statin and NSLLM use showed protective associations, with the strongest risk reductions observed when compared with GM users. Specifically, statin users had a 48\% lower risk (HR 0.52, 95\% CI: 0.40-0.68), and NSLLM users a 43\% lower risk (HR 0.57, 95\% CI: 0.41-0.79), of metastatic PC compared with GM users. Furthermore, statin users had a 49\% lower risk (HR 0.51, 95\% CI: 0.41-0.63), and NSLLM users a 34\% lower risk of PC mortality (HR 0.66, 95\% CI: 0.51-0.85). In sensitivity analyses, survival models accounting for competing risks were run, but results were identical. Moreover, when the NSLLM group and their comparators (non-users and GM users) were revised to remove men who also used statins, representing "pure" NSLLM users, the hazard ratios were similar, if not stronger, than the original results (Table 3). The magnitude of associations is depicted in Kaplan-Meier curves (Figures 1 and 2).

\section{Discussion}

We demonstrate that statins and NSLLM are potentially associated with a dramatic decrease in metastatic PC and PC mortality. The slight increase in PC incidence detected within the statin and NSLLM user cohorts may result from a real association or from extraneous factors such as greater exposure to health care than among non-users. In our analysis of PC incidence between LLM users and GM users, with presumably more similar health care access, the higher incidence of PC in the LLM user group falls away, and the difference between the groups is non-significant. Overall, our analysis corroborates, by demonstrating an effect of statins, and extends, by revealing the association with NSLLM, the observations of several independent groups-statin use is not strongly associated with PC incidence, but is associated with a consistently measured reduction in aggressive, lethal disease and mortality $[11-13,15,16,28,29]$.

A priori, there are two independent lines that suggest the prostate and PC may be altered by cholesterol exposure: 1) both a long [30] and recent [3,5-7] history of clinical and experimental observation supports the prostate as an organ that accumulates cholesterol, has difficulty removing excess cholesterol, and is physiologically sensitive to the effects of excess cholesterol; 2) a body of evidence suggests elevated circulating cholesterol is associated with risk of both benign and malignant prostate disease [12,31,32]. Therefore, we anticipated that statins and NSLLM would have a physiological effect on the prostate, alter the course of prostate pathology, and reduce the progression of PC. We hypothesized that LLM, and especially statins, whose major patient-relevant in vivo activity is to lower circulating LDL, would reduce incident metastatic PC and PC mortality. Our analysis of NSLLM and PC risk supports our hypothesis that lipid lowering affects prostate pathology and the progression of malignant disease; NSLLM had much the same effect on metastatic PC and PC mortality as did statins. To our knowledge, this is the first study to report that NSLLM also reduce the risk of clinically serious disease.

While the major in vivo effect of statins has always been acknowledged as their potent LDLcholesterol reducing activity, other activities that may augment statins' effectiveness have been elucidated, with some investigators/clinicians suggesting that LDL-cholesterol 
reduction, per se, is not why statins are so effective in reducing disease risk, whether CVD or serious PC. Recently, at least with regards to CVD, these non-cholesterol-basedmechanisms have been challenged by the publication of IMPROVE-IT trial results [33], in which ezetimibe (a NSLLM) in combination with a statin was more effective than a statin alone, and by results of studies using monoclonal antibodies targeting PCSK9, which leads to an increase in LDL-receptor expression (and lower circulating LDL-cholesterol). Because these drugs work by entirely different mechanisms than do statins, with their only similarity being that they reduce circulating cholesterol, the most reasonable argument is that statins, ezetimibe, and PCSK9 reduce disease because they reduce circulating cholesterol levels. Interestingly, in our analyses of NSLLM users, whether the men also used statins or not (i.e., "pure" NSLLM use) did not reduce protective associations with PC outcomes. Thus, our findings, suggesting that LDL-cholesterol reduction by any means reduces risk of serious PC, are very much in line with the consensus that LDL-cholesterol reduction reduces CVD risk [34].

The current report and several prior studies $[10,12-16,23,28]$ suggest that if statins influence PC risk, it is not readily apparent in a substantial reduction of incidence, but instead in reduction of aggressive, metastatic, or fatal disease. Why? First, cholesterol is not expected to be directly involved in altering genes and causing mutation, so an effect on incidence would not be anticipated. On the other hand, prostate ductal epithelial cells face a challenging environment when cholesterol levels are elevated, as excess cholesterol invites oxidation, which in turn can be cytotoxic [30], giving a selective advantage to cells capable of regulating excess cholesterol in the absence of appropriate export [35]. One way to accommodate the excess cholesterol is to convert cholesterol into other molecules such as bile acids and steroids [36]. Thus, a ductal epithelial cell in a high-cholesterol environment gains a selective advantage if it can carry on steroidogenesis, a process likely to be relatively unimportant to malignant cells when testosterone levels are high, but would be highly advantageous under castrate androgen levels, as would be encountered in late-stage disease. Steroidogenic potential might even provide certain cells with an aggressive phenotype when androgen levels are relatively normal.

As with use of all extant datasets, our study has strengths and unavoidable limitations. Strengths include a large sample of men and a rich dataset, with linked de-identified administrative health claims data, electronic prescription records, and cancer registry data for an entire Canadian province. Rather than relying on self-reported medication use, prescription records provided precise drug dispensing dates and dosage estimates [11]. In addition, a 25-year follow-up is sparse in prior studies. With such a large sample and long follow-up, there were sufficient events for statistical power. Utilizing the "active comparator, new user" design [22], with GM users as a reference population having similar investment in health care (e.g., need for physician visits and prescription refills), avoided many sources of bias. Lastly, the study's community-based sample provided more generalizability than a convenience sample or specialized study cohort $[16,28,29]$, and the study population's universal healthcare likely diminished socioeconomic biases related to health care access.

Counterbalancing limitations include the assumption that a dispensed prescription was a consumed prescription, lack of data on behavioral factors (e.g., body composition, dietary 
intake, and other conditions), which could help rule out unmeasured comorbidity as a potential contributor to mortality differences [25], possible misclassification of medical diagnoses and PC as the cause of mortality, and somewhat limited data on cancer progression and treatment. Further, the relatively homogeneous racial/ethnic population in Saskatchewan limits generalizability to other groups.

In summary, using a large patient population with prescription drug records spanning 25 years, this is the first study to demonstrate that lipid-lowering drugs (statins and non-statins) reduce the risk of aggressive PC and PC mortality. While our study cannot establish a causeand-effect relationship between cholesterol reduction and reduction in risk of serious PC outcomes, given that statins and NSLLMs work by entirely different non-overlapping mechanisms, a cholesterol-based explanation is simplest. Given the strength of the current observations and of many prior reports [3-7,10-16,24,28], it seems reasonable to inform men with established PC risk (e.g., family history, presence of BPH), that their use of statins and NSLLM could potentially reduce the possibility of a poor outcome if PC ever develops, and may therefore be beneficial.

\section{Supplementary Material}

Refer to Web version on PubMed Central for supplementary material.

\section{Acknowledgements}

The authors gratefully acknowledge the support of MaryRose Stang of the Population Health Branch, Saskatchewan Ministry of Health, and Michael Maurao, Weiqin Liao, and Ellen Cho of HealthCore-NERI.

Role of funding source

This work was supported by the National Institutes of Health National Institute on Aging (grant number R01AG038453). The National Institute on Aging had no role in study design, data collection, data analysis, data interpretation, or writing of the report. The content is solely the responsibility of the authors and does not necessarily represent the official views of the National Institutes of Health. This study is based in part on deidentified data provided by the Saskatchewan Ministry of Health. The interpretation and conclusions contained herein do not necessarily represent those of the Government of Saskatchewan or the Saskatchewan Ministry of Health.

Funding: This work was supported by the National Institutes of Health National Institute on Aging, Award Number R01AG038453

\section{References}

1. Cholesterol Treatment Trialists C, Kearney PM, Blackwell L, Collins R, Keech A, Simes J, et al. Efficacy of cholesterol-lowering therapy in 18,686 people with diabetes in 14 randomised trials of statins: a meta-analysis. Lancet 2008;371:117-25. [PubMed: 18191683]

2. Tonelli M, Lloyd A, Clement F, Conly J, Husereau D, Hemmelgarn B, et al. Efficacy of statins for primary prevention in people at low cardiovascular risk: a meta-analysis. CMAJ : Canadian Medical Association journal = journal de l'Association medicale canadienne 2011;183:E1189-202.

3. Pelton K, Freeman MR, Solomon KR. Cholesterol and prostate cancer. Curr Opin Pharmacol 2012;12:751-9. [PubMed: 22824430]

4. Solomon KR, Freeman MR. Do the cholesterol-lowering properties of statins affect cancer risk? Trends Endocrinol Metab 2008;19:113-21. [PubMed: 18356074]

5. Solomon KR, Freeman MR. The complex interplay between cholesterol and prostate malignancy. Urol Clin North Am 2011 ;38:243-59. [PubMed: 21798387] 
6. Solomon KR, Pelton K, Boucher K, Joo J, Tully C, Zurakowski D, et al. Ezetimibe is an inhibitor of tumor angiogenesis. Am J Pathol 2009;174:1017-26. [PubMed: 19179610]

7. Zhuang L, Kim J, Adam RM, Solomon KR, Freeman MR. Cholesterol targeting alters lipid raft composition and cell survival in prostate cancer cells and xenografts. J Clin Invest 2005; 115:959_ 68. [PubMed: 15776112]

8. Baigent C, Keech A, Kearney PM, Blackwell L, Buck G, Pollicino C, et al. Efficacy and safety of cholesterol-lowering treatment: prospective meta-analysis of data from 90,056 participants in 14 randomised trials of statins. Lancet 2005;366:1267-78. [PubMed: 16214597]

9. Browning DR, Martin RM. Statins and risk of cancer: a systematic review and metaanalysis. International journal of cancer 2007;120:833-43. [PubMed: 17131313]

10. Allott EH, Farnan L, Steck SE, Arab L, Su LJ, Mishel M, et al. Statin Use and Prostate Cancer Aggressiveness: Results from the Population-Based North Carolina-Louisiana Prostate Cancer Project. Cancer Epidemiol Biomarkers Prev 2016;25:670-7. [PubMed: 26819265]

11. Breau RH, Karnes RJ, Jacobson DJ, McGree ME, Jacobsen SJ, Nehra A, et al. The association between statin use and the diagnosis of prostate cancer in a population based cohort. The Journal of urology 2010;184:494-9. [PubMed: 20620405]

12. Farwell WR, D'Avolio LW, Scranton RE, Lawler EV, Gaziano JM. Statins and prostate cancer diagnosis and grade in a veterans population. Journal of the National Cancer Institute 2011;103:885-92. [PubMed: 21498780]

13. Jacobs EJ, Rodriguez C, Bain EB, Wang Y, Thun MJ, Calle EE. Cholesterol-lowering drugs and advanced prostate cancer incidence in a large U.S. cohort. Cancer Epidemiol Biomarkers Prev 2007;16:2213-7. [PubMed: 17971518]

14. Jespersen CG, Norgaard M, Friis S, Skriver C, Borre M. Statin use and risk of prostate cancer: a Danish population-based case-control study, 1997-2010. Cancer Epidemiol 2014;38:42-7. [PubMed: 24275259]

15. Murtola TJ, Tammela TL, Lahtela J, Auvinen A. Cholesterol-lowering drugs and prostate cancer risk: a population-based case-control study. Cancer Epidemiol Biomarkers Prev 2007;16:2226-32. [PubMed: 18006910]

16. Platz EA, Leitzmann MF, Visvanathan K, Rimm EB, Stampfer MJ, Willett WC, et al. Statin drugs and risk of advanced prostate cancer. Journal of the National Cancer Institute 2006;98:1819-25. [PubMed: 17179483]

17. Tan P, Zhang C, Wei SY, Tang Z, Gao L, Yang L, et al. Effect of statins type on incident prostate cancer risk: a meta-analysis and systematic review. Asian J Androl 2017;19:666-71. [PubMed: 27924788]

18. Chang CC, Ho SC, Chiu HF, Yang CY. Statins increase the risk of prostate cancer: a populationbased case-control study. The Prostate 2011;71:1818-24. [PubMed: 21480313]

19. Fowke JH, Motley SS, Barocas DA, Cookson MS, Concepcion R, Byerly S, et al. The associations between statin use and prostate cancer screening, prostate size, high-grade prostatic intraepithelial neoplasia (PIN), and prostate cancer. Cancer Causes Control 2011;22:417-26. [PubMed: 21170754]

20. Emilsson L, Garcia-Albeniz X, Logan RW, Caniglia EC, Kalager M, Hernan MA. Examining Bias in Studies of Statin Treatment and Survival in Patients With Cancer. JAMA oncology 2018;4:6370. [PubMed: 28822996]

21. Meng Y, Liao YB, Xu P, Wei WR, Wang J. Statin use and mortality of patients with prostate cancer: a meta-analysis. Onco Targets Ther 2016;9:1689-96. [PubMed: 27051303]

22. Lund JL, Richardson DB, Sturmer T. The active comparator, new user study design in pharmacoepidemiology: historical foundations and contemporary application. Current epidemiology reports 2015;2:221-8. [PubMed: 26954351]

23. Kantor ED, Lipworth L, Fowke JH, Giovannucci EL, Mucci LA, Signorello LB. Statin use and risk of prostate cancer: Results from the Southern Community Cohort Study. The Prostate 2015;75:1384-93. [PubMed: 26012482]

24. Mucci LA, Kantoff PW. Is the Evidence Sufficient to Recommend Statins for All Men With Prostate Cancer? Journal of clinical oncology : official journal of the American Society of Clinical Oncology 2017;35:3272-4. [PubMed: 28817369] 
25. Glynn RJ, Knight EL, Levin R, Avorn J. Paradoxical relations of drug treatment with mortality in older persons. Epidemiology 2001;12:682-9. [PubMed: 11679797]

26. Schneeweiss S, Patrick AR, Sturmer T, Brookhart MA, Avorn J, Maclure M, et al. Increasing levels of restriction in pharmacoepidemiologic database studies of elderly and comparison with randomized trial results. Medical care 2007;45:S131-42. [PubMed: 17909372]

27. Velentgas P, Dreyer NA, Nourjah P, Smith SR, Torchia MM, eds. Developing a Protocol for Observational Comparative Effectiveness Research: A User's Guide. AHRQ Publication No. 12(13)-EHC099. Rockville, MD: 2013 January 2013 Report No.

28. Tan N, Klein EA, Li J, Moussa AS, Jones JS. Statin use and risk of prostate cancer in a population of men who underwent biopsy. The Journal of urology 2011; 186:86-90. [PubMed: 21571344]

29. Flick ED, Habel LA, Chan KA, Van Den Eeden SK, Quinn VP, Haque R, et al. Statin use and risk of prostate cancer in the California Men's Health Study cohort. Cancer Epidemiol Biomarkers Prev 2007;16:2218-25. [PubMed: 17971519]

30. Schaffner CP. Prostatic cholesterol metabolism: Regulation and alteration In: Alan R, editor. The Prostatic Cell: Structure and Function. New York, NY: Liss, Inc; 1981 p. 279-324.

31. Platz EA, Till C, Goodman PJ, Parnes HL, Figg WD, Albanes D, et al. Men with low serum cholesterol have a lower risk of high-grade prostate cancer in the placebo arm of the prostate cancer prevention trial. Cancer Epidemiol Biomarkers Prev 2009;18:2807-13. [PubMed: 19887582]

32. Schnoeller TJ, Jentzmik F, Schrader AJ, Steinestel J. Influence of serum cholesterol level and statin treatment on prostate cancer aggressiveness. Oncotarget 2017;8:47110-20. [PubMed: 28445145]

33. Cannon CP, Blazing MA, Giugliano RP, McCagg A, White JA, Theroux P, et al. Ezetimibe Added to Statin Therapy after Acute Coronary Syndromes. The New England journal of medicine 2015;372:2387-97. [PubMed: 26039521]

34. Ference BA, Ginsberg HN, Graham I, Ray KK, Packard CJ, Bruckert E, et al. Low-density lipoproteins cause atherosclerotic cardiovascular disease. 1. Evidence from genetic, epidemiologic, and clinical studies. A consensus statement from the European Atherosclerosis Society Consensus Panel. European heart journal 2017;38:2459-72. [PubMed: 28444290]

35. Lee BH, Taylor MG, Robinet P, Smith JD, Schweitzer J, Sehayek E, et al. Dysregulation of cholesterol homeostasis in human prostate cancer through loss of ABCA1. Cancer Res 2013;73:1211-8. [PubMed: 23233737]

36. Mostaghel EA, Solomon KR, Pelton K, Freeman MR, Montgomery RB. Impact of circulating cholesterol levels on growth and intratumoral androgen concentration of prostate tumors. PLoS One 2012;7:e30062. [PubMed: 22279565] 


\section{Highlights}

- Statin users had lower risk of metastatic prostate cancer (PC) and PC mortality

- $\quad$ Non-statin lipid-lowering medication use showed similar associations as statins

- $\quad$ Cholesterol lowering seems to be the protective mechanism against advanced PC 
(A) Statin Users vs. Non-users
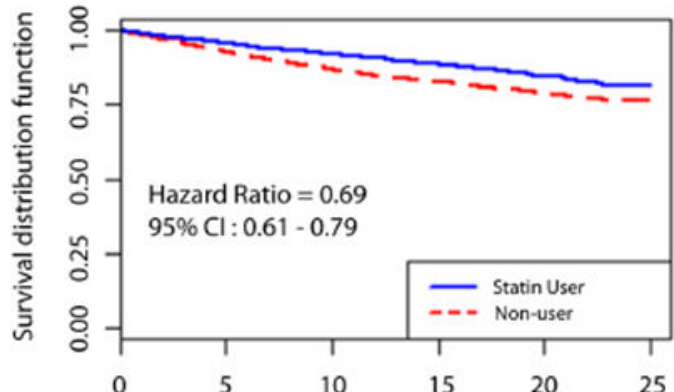

Person time in Years

Number at risk Statin users Non-users

Number at risk

Statin users GM users

$\begin{array}{rrrrrr}3157 & 2720 & 1868 & 887 & 320 & 0 \\ 5769 & 4669 & 2810 & 1149 & 388 & 0\end{array}$

(C) Statin Users vs. GM Users

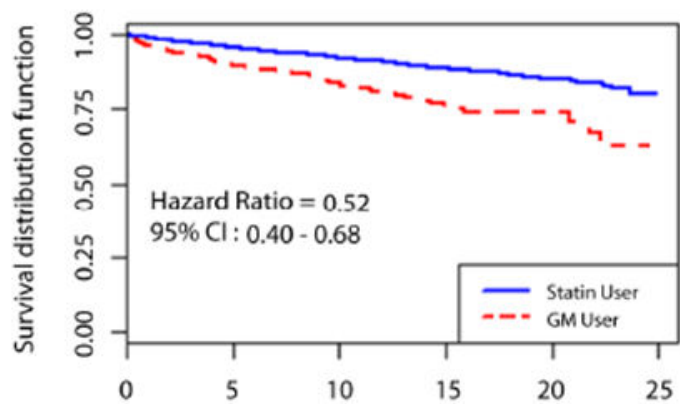

Person time in Years

$\begin{array}{rrrrrr}3016 & 2600 & 1791 & 840 & 293 & 0 \\ 416 & 334 & 201 & 102 & 27 & 0\end{array}$

Figure 1.
(B) NSLLM Users vs. Non-users

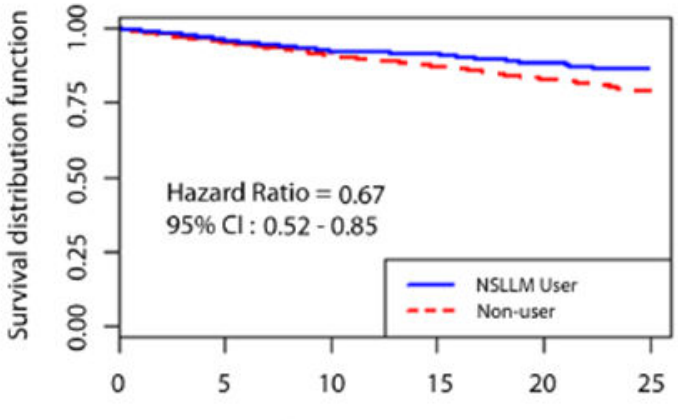

Person time in Years

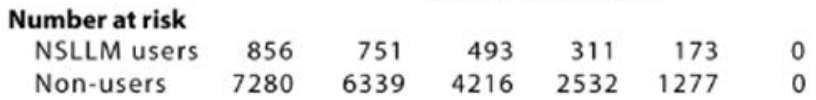

(D) NSLLM Users vs. GM Users

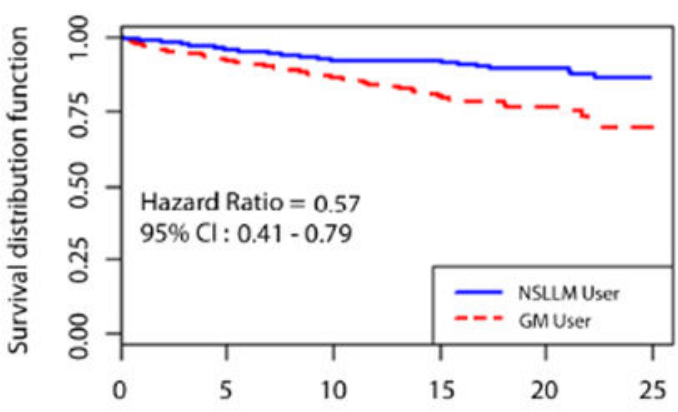

Person time in Years

\begin{tabular}{lrrrrrr} 
Number at risk & \multicolumn{8}{c}{ Person time in } \\
NSLLM users & 766 & 667 & 435 & 280 & 153 & 0 \\
GM users & 640 & 539 & 347 & 183 & 60 & 0
\end{tabular}

Kaplan-Meier curves for metastatic prostate cancer at diagnosis among statin and non-statin lipid-lowering medication (NSLLM) users compared with age-matched non-users (A and B, respectively) and glaucoma medication (GM) users ( $\mathrm{C}$ and $\mathrm{D}$, respectively). 
(A) Statin Users vs. Non-users

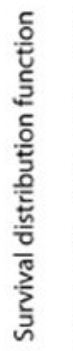

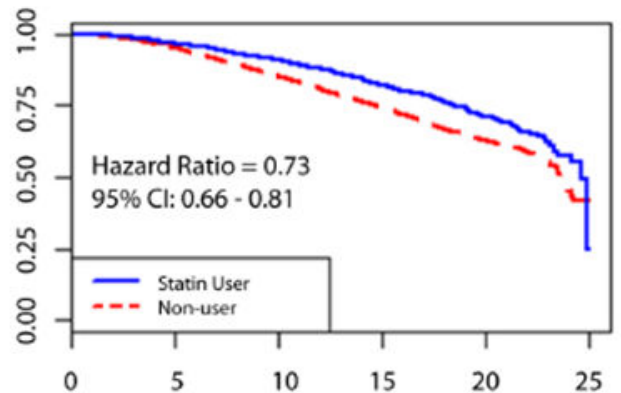

Number at risk Statin users Non-users

Number at risk Statin users GM users
Person time in Years

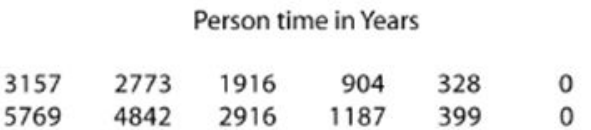

(C) Statin Users vs. GM Users

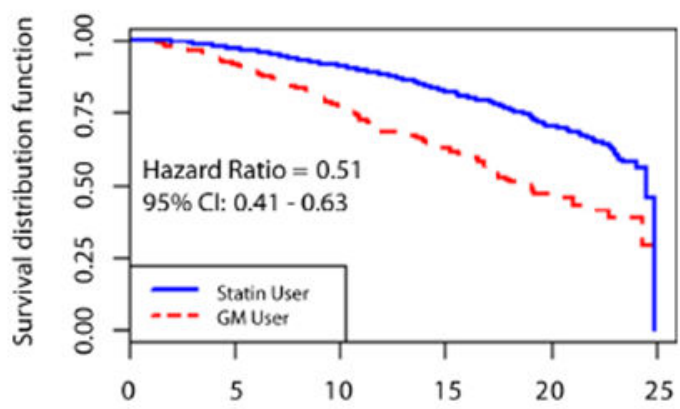

Person time in Years

$\begin{array}{rrrrrr}3016 & 2650 & 1837 & 856 & 300 & 0 \\ 416 & 346 & 211 & 109 & 28 & 0\end{array}$

Figure 2.
(B) NSLLM Users vs. Non-users

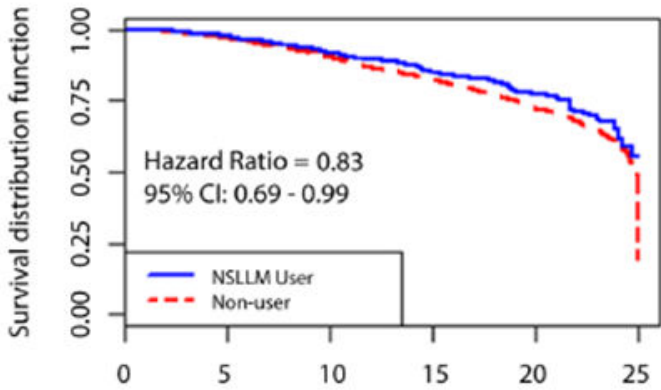

Person time in Years

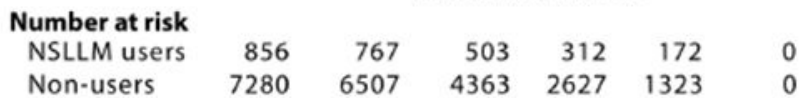

(D) NSLLM Users vs. GM Users

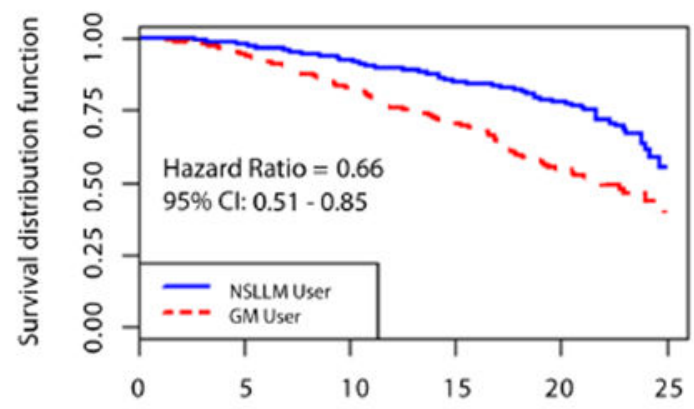

Person time in Years

Number at risk

NSLLM users

GM users

$\begin{array}{rrrrrr}766 & 682 & 444 & 280 & 153 & 0 \\ 640 & 556 & 363 & 196 & 63 & 0\end{array}$

Kaplan-Meier curves for prostate cancer mortality among statin and non-statin lipidlowering medication (NSLLM) users compared with age-matched non-users (A and B, respectively) and glaucoma medication (GM) users (C and D, respectively). 
Table 1.

Characteristics of male new users of statins, non-users, and glaucoma medication users

\begin{tabular}{|c|c|c|c|}
\hline Characteristic & Statin user ${ }^{\dagger}$ & Statin non-user & Glaucoma medication user \\
\hline $\mathrm{N}$ & 80,282 & 184,538 & 7,331 \\
\hline Age at index (yrs), Mean (SD) & $59.1(10.6)$ & $58.7(10.7)$ & $64.0(10.2)$ \\
\hline \multicolumn{4}{|l|}{ Year of index, $\mathrm{n}(\%)$} \\
\hline 1990-1999 & $13927(17.4 \%)$ & $27525(14.9 \%)$ & $3685(50.3 \%)$ \\
\hline 2000-2004 & $19171(23.9 \%)$ & $39089(21.2 \%)$ & $1221(16.7 \%)$ \\
\hline 2005-2009 & $25425(31.7 \%)$ & $58649(31.8 \%)$ & $1181(16.1 \%)$ \\
\hline 2010-2014 & $21759(27.1 \%)$ & $59275(32.1 \%)$ & $1244(17.0 \%)$ \\
\hline Follow-up time (yrs), Median (IQR) & $7.0(7.5)$ & $5.9(7.0)$ & $6.6(8.3)$ \\
\hline Age at exit (yrs), Mean (SD) & $66.6(11.2)$ & $65.2(11.2)$ & $71.7(10.8)$ \\
\hline \multicolumn{4}{|l|}{ Baseline drug use, $\mathrm{n}(\%)$} \\
\hline Non-statin lipid-lowering medications & $5802(7.2 \%)$ & $3165(1.7 \%)$ & $132(1.8 \%)$ \\
\hline Glaucoma medications & $2808(3.5 \%)$ & $6495(3.5 \%)$ & - \\
\hline 5a-reductase inhibitors & $1599(2.0 \%)$ & $4928(2.7 \%)$ & $115(1.6 \%)$ \\
\hline a-blockers & $6781(8.5 \%)$ & $17953(9.7 \%)$ & $496(6.8 \%)$ \\
\hline \multicolumn{4}{|l|}{ Baseline comorbidities, n (\%) } \\
\hline Benign prostatic hyperplasia & $8614(10.7 \%)$ & $23030(12.5 \%)$ & $951(13.0 \%)$ \\
\hline Diabetes & $20081(25.0 \%)$ & $16438(8.9 \%)$ & $949(13.0 \%)$ \\
\hline Hypertension & $39845(30.3 \%)$ & $62665(34.0 \%)$ & $2451(33.4 \%)$ \\
\hline Heart disease & $33082(41.2 \%)$ & $37644(20.4 \%)$ & $1854(25.3 \%)$ \\
\hline Hyperlipidemia ${ }^{t}$ & $39825(49.6 \%)$ & $26553(14.4 \%)$ & $668(9.1 \%)$ \\
\hline \multicolumn{4}{|l|}{ Outcomes during follow-up, $\mathrm{n}(\%)$} \\
\hline Prostate cancer (PC) & $3157(3.9 \%)$ & $5769(3.1 \%)$ & $416(5.7 \%)$ \\
\hline Metastatic PC at diagnosis & $307(0.4 \%)$ & $788(0.4 \%)$ & $78(1.1 \%)$ \\
\hline \multicolumn{4}{|l|}{ Mortality among men with PC } \\
\hline Prostate cancer & $497(0.6 \%)$ & $1138(0.6 \%)$ & $135(1.8 \%)$ \\
\hline Other cancer & $164(0.2 \%)$ & $378(0.2 \%)$ & $57(0.8 \%)$ \\
\hline Non-cancer & $477(0.6 \%)$ & $748(0.4 \%)$ & $109(1.5 \%)$ \\
\hline Alive & $2019(2.5 \%)$ & $3505(1.9 \%)$ & $115(1.6 \%)$ \\
\hline
\end{tabular}

${ }^{\dagger}$ In comparing statin users with glaucoma medication users, users of both drugs were removed, reducing the statin user sample size to $77,453$.

${ }^{*}$ ICD-9 272 is not specific to hyperlipidemia (includes other disorders of lipoid metabolism). 
Table 2.

Characteristics of male new users of non-statin lipid-lowering medications (NSLLM), non-users, and glaucoma medication users

\begin{tabular}{|c|c|c|c|}
\hline Characteristic & NSLLM user ${ }^{\dagger}$ & NSLLM non-user & Glaucoma medication user \\
\hline $\mathrm{N}$ & 20,251 & 185,589 & 11,203 \\
\hline Age at index (yrs), Mean (SD) & $58.7(10.3)$ & $58.2(10.1)$ & $64.4(10.2)$ \\
\hline \multicolumn{4}{|l|}{ Year of index, $\mathrm{n}(\%)$} \\
\hline 1990-1999 & $4981(24.6 \%)$ & $44748(24.1 \%)$ & $4885(43.3 \%)$ \\
\hline 2000-2004 & $3623(17.9 \%)$ & $32578(17.6 \%)$ & $2059(18.4 \%)$ \\
\hline 2005-2009 & $6693(33.1 \%)$ & $61286(33.0 \%)$ & $2164(19.3 \%)$ \\
\hline 2010-2014 & $4954(24.5 \%)$ & $46977(25.3 \%)$ & $2125(19.0 \%)$ \\
\hline Follow-up time (yrs), Median (IQR) & $7.3(7.5)$ & $7.1(7.5)$ & $7.3(8.8)$ \\
\hline Age at exit (yrs), Mean (SD) & $66.8(10.8)$ & $66.2(10.8)$ & $72.4(10.6)$ \\
\hline \multicolumn{4}{|l|}{ Baseline drug use, $\mathrm{n}(\%)$} \\
\hline Statins & $11807(58.3 \%)$ & $38134(20.6 \%)$ & $1817(16.2 \%)$ \\
\hline Glaucoma medications & $716(3.5 \%)$ & $6450(3.5 \%)$ & - \\
\hline 5a-reductase inhibitors & $446(2.2 \%)$ & $4130(2.2 \%)$ & $226(2.0 \%)$ \\
\hline a-blockers & $1914(9.5 \%)$ & $17077(9.2 \%)$ & $991(8.9 \%)$ \\
\hline \multicolumn{4}{|l|}{ Baseline comorbidities, $\mathrm{n}(\%)$} \\
\hline Benign prostatic hyperplasia & $2349(11.6 \%)$ & $21846(11.8 \%)$ & $1622(14.5 \%)$ \\
\hline Diabetes & $5716(28.2 \%)$ & $26243(14.1 \%)$ & $2143(19.1 \%)$ \\
\hline Hypertension & $11417(56.4 \%)$ & $72335(39.0 \%)$ & $4597(41.0 \%)$ \\
\hline Heart disease & $8989(44.4 \%)$ & $47089(25.4 \%)$ & $3421(30.5 \%)$ \\
\hline Hyperlipidemia & $13491(66.6 \%)$ & $46690(25.2 \%)$ & $2123(19.0 \%)$ \\
\hline \multicolumn{4}{|l|}{ Outcomes during follow-up, $\mathrm{n}(\%)$} \\
\hline Prostate cancer (PC), n (\%) & $856(4.2 \%)$ & $7280(3.9 \%)$ & $640(5.7 \%)$ \\
\hline Metastatic PC at diagnosis & $72(0.4 \%)$ & $854(0.5 \%)$ & $106(1.0 \%)$ \\
\hline \multicolumn{4}{|l|}{ Mortality among men with PC } \\
\hline Prostate cancer & $129(0.6 \%)$ & $1330(0.7 \%)$ & $179(1.6 \%)$ \\
\hline Other cancer & $48(0.2 \%)$ & $424(0.2 \%)$ & $66(0.6 \%)$ \\
\hline Non-cancer & $153(0.8 \%)$ & $1004(0.5 \%)$ & $155(1.4 \%)$ \\
\hline Alive & $526(2.6 \%)$ & $4552(2.4 \%)$ & $240(2.1 \%)$ \\
\hline
\end{tabular}

${ }^{\dagger}$ In comparing NSLLM users with glaucoma medication users, users of both drugs were removed, reducing the NSLLM user sample size to 18,865 .

ICD-9 272 is not specific to hyperlipidemia (includes other disorders of lipoid metabolism). 
Table 3.

Risk of prostate cancer, metastatic prostate cancer, and prostate cancer mortality among statin users, non-statin lipid-lowering medication (NSLLM) users, non-users, and glaucoma medication users

\begin{tabular}{|c|c|c|c|c|c|}
\hline & No. of events & Age-adjusted HR (95\% CI) & P value & Fully-adjusted $\mathrm{HR}^{\dagger}(95 \% \mathrm{CI})$ & P value \\
\hline \multicolumn{6}{|c|}{ Statin Users $(\mathrm{n}=80,282)$ vs. Non-users $(\mathrm{n}=184,538)$} \\
\hline Total prostate cancer & 8926 & $1.06(1.01-1.11)$ & 0.009 & $1.07(1.02-1.12)$ & 0.003 \\
\hline Metastatic prostate cancer & 1095 & $0.67(0.59-0.77)$ & $<0.0001$ & $0.69(0.61-0.79)$ & $<0.0001$ \\
\hline Prostate cancer mortality & 1635 & $0.71(0.64-0.79)$ & $<0.0001$ & $0.73(0.66-0.81)$ & $<0.0001$ \\
\hline \multicolumn{6}{|c|}{ NSLLM Users $(\mathrm{n}=20,251)$ vs. Non-users $(\mathrm{n}=185,589)$} \\
\hline Total prostate cancer & 8136 & $1.05(0.98-1.13)$ & 0.16 & $1.14(1.06-1.22)$ & 0.0006 \\
\hline Metastatic prostate cancer & 926 & $0.69(0.54-0.87)$ & 0.002 & $0.67(0.52-0.85)$ & 0.001 \\
\hline Prostate cancer mortality & 1459 & $0.79(0.66-0.95)$ & 0.01 & $0.83(0.69-0.99)$ & 0.04 \\
\hline \multicolumn{6}{|c|}{ "Pure" NSLLM Users $(\mathrm{n}=8,444)$ vs. Non-users $(\mathrm{n}=147,455)^{\not t}$} \\
\hline Total prostate cancer & 6614 & $1.04(0.95-1.15)$ & 0.41 & $1.02(0.93-1.12)$ & 0.68 \\
\hline Metastatic prostate cancer & 739 & $0.61(0.43-0.85)$ & 0.004 & $0.63(0.45-0.89)$ & 0.008 \\
\hline Prostate cancer mortality & 1259 & $0.75(0.60-0.94)$ & 0.01 & $0.77(0.62-0.97)$ & 0.02 \\
\hline \multicolumn{6}{|c|}{ Statin Users $(\mathrm{n}=77,453)$ vs. Glaucoma Medication Users $(\mathrm{n}=7,331)$} \\
\hline Total prostate cancer & 3432 & $0.87(0.78-0.97)$ & 0.009 & $1.02(0.92-1.14)$ & 0.71 \\
\hline Metastatic prostate cancer & 362 & $0.58(0.45-0.75)$ & $<0.0001$ & $0.52(0.40-0.68)$ & $<0.0001$ \\
\hline Prostate cancer mortality & 603 & $0.58(0.47-0.70)$ & $<0.0001$ & $0.51(0.41-0.63)$ & $<0.0001$ \\
\hline \multicolumn{6}{|c|}{ NSLLM Users $(\mathrm{n}=18,865)$ vs. Glaucoma Medication Users $(\mathrm{n}=11,203)$} \\
\hline Total prostate cancer & 1406 & $0.97(0.87-1.08)$ & 0.59 & $1.08(0.95-1.22)$ & 0.24 \\
\hline Metastatic prostate cancer & 168 & $0.62(0.45-0.86)$ & 0.004 & $0.57(0.41-0.79)$ & 0.0008 \\
\hline Prostate cancer mortality & 292 & $0.68(0.54-0.87)$ & 0.002 & $0.66(0.51-0.85)$ & 0.001 \\
\hline \multicolumn{6}{|c|}{ "Pure" NSLLM Users $(\mathrm{n}=7,816)$ vs. Glaucoma Medication Users $(\mathrm{n}=9,386)^{*}$} \\
\hline Total prostate cancer & 986 & $1.06(0.93-1.22)$ & 0.39 & $1.10(0.96-1.26)$ & 0.18 \\
\hline Metastatic prostate cancer & 130 & $0.53(0.34-0.80)$ & 0.003 & $0.50(0.33-0.76)$ & 0.001 \\
\hline Prostate cancer mortality & 235 & $0.66(0.49-0.87)$ & 0.004 & $0.65(0.49-0.87)$ & 0.003 \\
\hline
\end{tabular}

